\title{
DISCOVERY OF NEW, DUST-POOR B[e] SUPERGIANTS IN THE SMALL MAGELLANIC CLOUD
}

\author{
A. S. Graus, J. B. Lamb, and M. S. Oey \\ Department of Astronomy, University of Michigan, 830 Dennison Building, Ann Arbor, MI 48109-1042, USA; asgraus@umich.edu \\ Received 2012 June 7; accepted 2012 September 10; published 2012 October 11
}

\begin{abstract}
We present the discovery of three new B[e] supergiants ( $\operatorname{sgB}[\mathrm{e}]$ stars) in the Small Magellanic Cloud (SMC). All three stars (R15, R38, and R48) were identified in the course of our Runaways and Isolated O Type Star Spectroscopic Survey of the SMC. The stars show optical spectra that closely resemble those of previously known $\mathrm{B}[\mathrm{e}]$ stars, presenting numerous low-ionization forbidden and permitted emission lines such as [Fe II] and Fe II. Furthermore, our stars have luminosities of $\log \left(L / L_{\odot}\right) \geqslant 4$, demonstrating that they are supergiants. However, we find lower infrared excesses and weaker forbidden emission lines than for previously identified $\mathrm{B}[\mathrm{e}]$ supergiants. Thus our stars appear either to have less material in their circumstellar disks than other $\operatorname{sgB}[\mathrm{e}]$ stars, or the circumstellar material has lower dust content. We suggest that these may constitute a new subclass of dust-poor $\operatorname{sgB}[\mathrm{e}] \mathrm{stars}$.
\end{abstract}

Key words: circumstellar matter - infrared: stars - Magellanic Clouds - stars: emission-line, Be - stars: evolution - stars: winds, outflows

Online-only material: color figure

\section{INTRODUCTION}

Massive stars comprise a small fraction of the total number of stars in the universe and are short-lived. However, they provide a dominant portion of the mechanical and radiative energy of galaxies. Massive stars can enrich the universe with metals, trigger star formation, and are a potential source for the reionization of the universe. The evolution of massive stars is central to their ability to influence their surroundings. Therefore, a better understanding of the phases of massive stellar evolution will help us comprehend many physical processes that take place within galaxies.

In massive stars, the $\mathrm{B}[\mathrm{e}]$ phenomenon is an evolutionary phase whose origin remains unclear. Stars exhibiting the $\mathrm{B}[\mathrm{e}]$ phenomenon show numerous emission lines, and normally show infrared radiation from dust. These properties point to the presence of large amounts of circumstellar material, most likely in a disk configuration. The $\mathrm{B}[\mathrm{e}]$ phenomenon is perplexing because stars in many different stages of evolution can exhibit its signs. Lamers et al. (1998) divided the types of stars showing the $\mathrm{B}[\mathrm{e}]$ phenomenon into five groups: post-main-sequence $\mathrm{B}[\mathrm{e}]$ supergiants ( $\mathrm{sgB}[\mathrm{e}]$ stars), pre-main-sequence Herbig $\mathrm{B}[\mathrm{e}]$ stars, compact planetary nebulae, symbiotic $\mathrm{B}[\mathrm{e}]$ stars, and an entire category of unclassified B [e] stars. A link between strong infrared excess and B stars showing forbidden and permitted emission lines was identified by Geisel (1970) and Allen \& Swings (1976). Conti (1976) then suggested that "B-type stars which show forbidden emission lines" should be classified as $\mathrm{B}[\mathrm{e}]$ stars. Since then, many more B[e] stars have been found, leading to a standard definition of the $\mathrm{B}[\mathrm{e}]$ phenomenon as follows (e.g., Zickgraf 1998; Lamers et al. 1998): strong Balmer emission lines, low-excitation permitted and forbidden emission lines from low-ionization metals such as $\mathrm{Fe}$, and a strong excess in the near- or mid-infrared due to circumstellar dust. Among the more homogeneous and well-studied group of stars showing the $\mathrm{B}[\mathrm{e}]$ phenomenon are the $\mathrm{B}[\mathrm{e}]$ supergiants.

Supergiant $\mathrm{B}[\mathrm{e}]$ stars $(\mathrm{sgB}[\mathrm{e}])$ also have well-defined criteria laid out by Lamers et al. (1998), including supergiant luminosity $\left(\log L / L_{\odot} \geqslant 4\right)$, indicators of evolution off the main sequence, broad ultraviolet absorption lines, and signs of nitrogen abundance enhancement. In order to explain the emission properties of $\mathrm{sgB}[\mathrm{e}]$ stars, Zickgraf et al. (1985) proposed a twocomponent wind model where the star is rotating near break-up velocity and simultaneously close to the Eddington limit. This causes the star to emit a wind that is fast and sparse near the poles, producing high-excitation absorption, while being denser around the equator, producing the emission features. The twocomponent wind allows for the creation of a dusty circumstellar disk, which produces the infrared excess seen around $\operatorname{sgB}[\mathrm{e}]$ stars. In this scenario, the star begins exhibiting the $\mathrm{B}[\mathrm{e}]$ phenomenon sometime after the red supergiant phase, but before He-core burning begins (Lamers 2006). Some sgB[e] stars exhibit properties akin to those of luminous blue variables (LBVs), suggesting that $\mathrm{LBVs}$ and at least some $\mathrm{sgB}[\mathrm{e}]$ stars are related, although their relation remains unclear, as summarized by Lamers (2006). R4 in the Small Magellanic Cloud (SMC) is a prominent candidate for this model. R4 is currently classified as an $\mathrm{LBV} / \mathrm{sgB}[\mathrm{e}]$ because it shows properties of both objects. Pasquali et al. (2000) further suggested that R4 could have formed in a binary merger. It is unknown whether this is a common origin for $\mathrm{sgB}[\mathrm{e}]$ stars in general, or whether $\mathrm{R} 4$ is a unique case. Others have suggested that $\mathrm{sgB}[\mathrm{e}]$ stars arise from interacting binaries, which is consistent with discoveries of binary companions around $\operatorname{sgB}[\mathrm{e}]$ stars (e.g., Wheelwright et al. 2012; Miroshnichenko et al. 2003). Binary companions have been found around other types of $\mathrm{B}[\mathrm{e}]$ stars as well, such as around Herbig B[e] stars (e.g., Kraus et al. 2012). This scenario could help explain why stars exhibiting the $\mathrm{B}[\mathrm{e}]$ phenomenon show similar properties while apparently being at different stages of evolution.

In this paper, we report the discovery of three previously unidentified $\mathrm{sgB}$ [e] stars in the SMC: R15, R38, and R48 (Feast et al. 1960), along with observations of a previously known sgB[e], AzV 154 (Azzopardi et al. 1975), obtained from the Runaways and Isolated O Star Spectroscopic Survey of the SMC (RIOTS4; Oey \& Lamb 2011; Lamb et al. 2011). The RIOTS4 survey is a photometrically selected and spatially complete spectroscopic survey of OB stars in the SMC. The three previously unknown $\mathrm{sgB}[\mathrm{e}]$ stars all show optical spectra characteristic of the $\mathrm{B}[\mathrm{e}]$ phenomenon, including forbidden and 


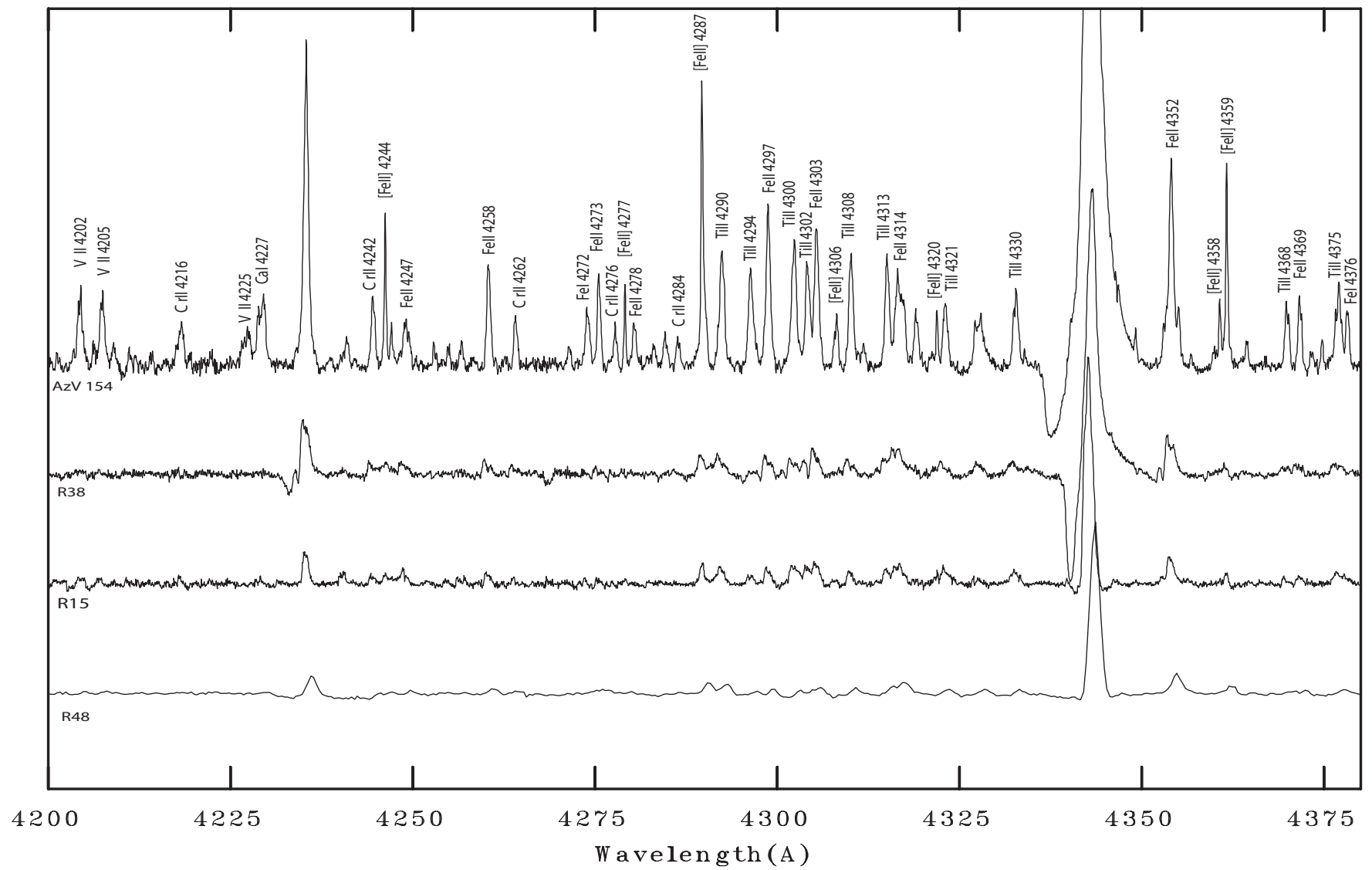

Figure 1. Optical spectra of the region near Fe II $\lambda 4233$ and $\mathrm{H} \gamma$, for the previously known sgB[e] star AzV 154, along with those of the newly discovered sgB[e] stars R38, R15, and R48 (top to bottom). The top three spectra were obtained with the MIKE echelle spectrograph, and the bottom spectrum with IMACS.

permitted emission. These stars also show additional indicators that they are supergiants, and they appear to be post-mainsequence stars.

\section{RIOTS4 DATA}

The RIOTS4 survey was carried out for a photometrically selected sample of field OB stars in the SMC (Oey \& Lamb 2011; Lamb et al. 2011). OB stars were selected from a $U B V R$ photometric survey of the SMC carried out by Massey (2002). The most massive $\mathrm{O}$ stars, along with $\mathrm{B}$ stars of roughly type B1.5 and earlier, were selected from these data by requiring that $B \leqslant 15.21$ and the reddening free parameter $Q_{\mathrm{UBR}} \leqslant-0.84$. Oey et al. (2004) then identified field stars in this sample with the use of a friends-of-friends algorithm (Battinelli 1991). Stars were considered clustered if another massive star was found within a given clustering length of the target star. For this survey the clustering length was 28 pc. The field stars selected by the friends-of-friends algorithm were then observed using the Inamori Magellan Areal Camera and Spectrograph (IMACS) and Magellan Inamori Kyocera Echelle (MIKE) spectrograph on the Magellan telescopes at Las Campanas Observatory.

Over the course of the survey we discovered four stars showing rich emission-line spectra: R38, R48, R15, and AzV 154. Of these stars, AzV154 is a known sgB[e] (Zickgraf et al. 1989). R48 was observed using the IMACS multi-slit mode on the Magellan Baade telescope, using the $f / 4$ camera with a 1200 lines $\mathrm{mm}^{-1}$ grating and a 0 !'7 slit, yielding a spectral resolution of $R \sim 3600$, and a wavelength range of 3650-5250 $\mathrm{A}$. $\mathrm{R} 38$, $\mathrm{R} 15$, and AzV 154 were observed with the MIKE echelle spectrograph on the Magellan Clay telescope. These observations were made using a $1^{\prime \prime}$ slit, yielding a resolution of $R \sim 28,000$, and a wavelength range of 3250-5050 ̊.

For the IMACS data, the reduction, bias subtraction, wavelength calibration, and flat fielding of the two-dimensional spectra were performed using the $\operatorname{COSMOS}^{1}$ data reduction pipeline. The spectrum was extracted using the apextract package in IRAF. $^{2}$ For data obtained using the MIKE spectrograph, the data were reduced and wavelength-calibrated using IRAF packages. The one-dimensional spectra from both instruments were then rectified and cleaned of cosmic rays and bad pixels using IRAF.

\section{OPTICAL PROPERTIES}

\subsection{Optical Spectra}

Optical spectra of our three newly identified $\mathrm{sgB}[\mathrm{e}]$ stars, along with that of the previously known object, are shown in Figure 1, and their properties are given in Table 1. Table 2 gives their photometry. Our optical spectra clearly show strong emission features, particularly in the Balmer series. R38 exhibits strong P Cygni profiles in its Balmer lines as does the previously known $\mathrm{sgB}[\mathrm{e}] \mathrm{star}, \mathrm{AzV} 154$, indicating intense mass loss. In accordance with the standard definition of the $\mathrm{B}[\mathrm{e}]$ phenomenon presented in, e.g., Zickgraf (1998) and Lamers et al. (1998), our stars also show numerous other emission lines. These lines are

\footnotetext{
1 http://code.obs.carnegiescience.edu/cosmos

2 IRAF is distributed by the National Optical Astronomy Observatories, which is operated by the Association of Universities for Research in Astronomy, Inc., under cooperative agreement with the National Science Foundation.
} 
Table 1

RIOTS4 SMC sgB[e] Stars

\begin{tabular}{|c|c|c|c|c|c|c|c|c|c|c|}
\hline Massey (2002) & $A z V^{a}$ & Radcliffe $^{\text {b }}$ & R.A. (J2000) & Decl. & $\mathrm{SpT}^{\mathrm{c}}$ & $\log \left(L / L_{\odot}\right)$ & $v \sin i^{\mathrm{d}}$ & $\mathrm{RV}^{\mathrm{d}}$ & $\left\langle v_{\mathrm{rms}}\right\rangle^{\mathrm{d}}$ & $\left\langle v_{\mathrm{fb}, \mathrm{rms}}\right\rangle^{\mathrm{d}}$ \\
\hline 29267 & AzV 154 & $\ldots$ & $00: 54: 09.52$ & $-72: 41: 43.3$ & B0 I & $5.23 \pm 0.15$ & $\ldots$ & 141.0 & 3.1 & 3.4 \\
\hline 46398 & AzV 230 & $\mathrm{R} 15$ & $00: 59: 29.20$ & $-72: 01: 04.6$ & B7 I & $4.79 \pm 0.17$ & 56 & 126.4 & 9.9 & 16.6 \\
\hline 62661 & AzV 390 & R38 & 01:05:47.01 & $-71: 46: 21.9$ & B8 I & $4.61 \pm 0.15$ & 53 & 105.0 & 7.3 & 15.2 \\
\hline 83480 & $\ldots$ & $\mathrm{R} 48$ & $01: 30: 10.89$ & $-73: 18: 56.2$ & B6 I & $4.36 \pm 0.12$ & 108 & 202.5 & 10.0 & 13.9 \\
\hline
\end{tabular}

Notes.

a Identification from Azzopardi et al. (1975).

b Identification from Feast et al. (1960).

c Spectral type for AzV 154 is from Zickgraf et al. (1989); those for R15, R48, and R38 are new.

${ }^{\mathrm{d}} \mathrm{km} \mathrm{s}^{-1}$.

Table 2

Photometry $^{\mathrm{a}}$

\begin{tabular}{|c|c|c|c|c|c|c|c|c|c|c|c|c|c|}
\hline Star & $A_{V}^{\mathrm{b}}$ & $U$ & $B$ & $V$ & $R$ & $J$ & $H$ & $K$ & {$[3.6]$} & {$[4.5]$} & {$[5.8]$} & {$[8.0]$} & [24] \\
\hline $\mathrm{AzV} 154$ & $0.24 \pm 0.21$ & 12.80 & 13.48 & 13.20 & 12.64 & 12.35 & 11.93 & 11.11 & 9.18 & 8.34 & 7.75 & 6.97 & 4.79 \\
\hline $\mathrm{R} 15$ & $0.17 \pm 0.21$ & 11.81 & 12.67 & 12.64 & 12.46 & 12.37 & 12.28 & 12.10 & 11.51 & 11.29 & 10.10 & 10.63 & 9.30 \\
\hline R38 & $0.16 \pm 0.18$ & 12.24 & 13.01 & 12.98 & 12.82 & 12.68 & 12.59 & 12.38 & 11.97 & 11.77 & 11.41 & 11.17 & $\ldots$ \\
\hline $\mathrm{R} 48$ & $0.34 \pm 0.26$ & 12.92 & 13.74 & 13.73 & 13.54 & 13.45 & 13.34 & 13.18 & 12.79 & 12.57 & 12.38 & 11.94 & $\ldots$ \\
\hline VFTS $698^{c}$ & $\ldots$ & $\ldots$ & 14.12 & 13.68 & $\ldots$ & 12.34 & 11.89 & 11.43 & 10.60 & 10.23 & 10.02 & 9.66 & $\ldots$ \\
\hline
\end{tabular}

Notes.

${ }^{a}$ UBVR photometry is taken from Massey (2002), JHK from 2MASS (Skrutskie et al. 2006), and 3.6-24 $\mu$ m from the SAGE SMC survey (Gordon et al. 2011).

b Visual extinction values taken from Zaritsky et al. (2002).

c Photometry for VFTS 698 from Dunstall et al. (2012).

primarily permitted and forbidden low-ionization lines from metals, such as Fe II, [Fe II], Ti II, Cr II, and V II. We identified emission lines in the spectral range 4000-4750 $\AA$, based on the work of Zickgraf et al. (1989), Jaschek \& Andrillat (1998), Andrillat et al. (1997), and Jaschek et al. (1996a, 1996b). Four to twelve forbidden emission lines are confirmed in each of the three new stars at these wavelengths, including [Fe II] $\lambda \lambda 4288,4360,4414$, and [Ni II] $\lambda 4462$, which are detected in all objects. We list the heliocentric radial velocities (RVs) in Table 1, together with the root-mean-square velocity residual $\left\langle v_{\text {rms }}\right\rangle$ measured for the fitted RV from both permitted and forbidden lines, as well as that measured from only the forbidden lines $\left\langle v_{\mathrm{fb}, \mathrm{rms}}\right\rangle$. Given that there are fewer forbidden lines, the values of $\left\langle v_{\mathrm{fb}, \mathrm{rms}}\right\rangle$ are fully consistent with those of $\left\langle v_{\mathrm{rms}}\right\rangle$.

The absorption lines of B-star spectra are also present, for example, $\mathrm{He}$ I, Si II, and Mg II. The classification criteria of Fitzpatrick (1991) and Walborn \& Fitzpatrick (1990) were used for spectral typing of our stars (Table 1). The presence in absorption of $\mathrm{Si}$ II $\lambda \lambda 4128-30$, and the relative strength of $\mathrm{Mg}$ II $\lambda 4481$ compared to He I $\lambda 4471$ indicate the spectral types of all three of our newly discovered $\mathrm{sgB}[\mathrm{e}]$ stars to be in the midto late-B range. These spectral types are unexpectedly cooler than those derived for normal OB stars in the RIOTS4 survey. While it is possible that a cooler binary companion dominates the optical spectra, it turns out that higher $R$-band fluxes caused by the strong $\mathrm{H} \alpha$ emission in the decretion disks actually lead to lower $Q_{\mathrm{UBR}}$ values, mimicking a bluer continuum. This reveals a selection bias for the RIOTS4 survey which thus includes other $R$-excess objects, in addition to the target blue stars, thereby also selecting a large number of classical Be stars.

We can use the photospheric absorption lines to estimate the projected rotational velocity of our stars (Table 1) by Gaussian fitting of the Si II, Mg II, and He I lines. The values given in the table are rough estimates that correspond directly to the measured FWHM, since the accurate measurement of $v \sin i$ is difficult; effects such as macroturbulence can cause significant, additional line broadening for supergiants (SimónDíaz \& Herrero 2007). Of course, another important factor is the inclination angle with which we are viewing our stars. Two of our stars (R15 and R38) show single-peaked Balmer emission lines. If the Balmer emission comes from a disk, single-peaked lines imply that we are viewing these two objects close to pole-on (Stee \& de Araujo 1994), so their rotational velocities could be significantly higher. This therefore suggests that macroturbulence significantly affects our measurements of the projected rotational velocity in Table 1 .

We calculated the stellar luminosities from their $V$-band fluxes, using photometry from Massey (2002; Table 2), along with bolometric corrections for B supergiants from Drilling \& Landolt (2000). These luminosities may be upper limits since there may be some contribution from the circumstellar disk. Photometry from the All Sky Automated Survey (Pojmanski 1997) hints that our three new $\operatorname{sgB}[\mathrm{e}]$ stars may be variable at a level of $\sim 0.1 \mathrm{mag}$ in $V$, and the known sgB[e] star AzV 154 shows variability in $V$ at a level of 0.25 mag. Lamers et al. (1998) note that $\mathrm{sgB}[\mathrm{e}]$ generally have low photometric variability. Extinction values were estimated using the extinction maps of Zaritsky et al. (2002), and a distance modulus of 18.9 was used (Harries et al. 2003). These quantities are also given in Table 2.

\subsection{Supergiant $B[e]$ or Herbig $B[e]$ ?}

We now use the data we have compiled to constrain the evolutionary state of our three newly discovered $\mathrm{B}[\mathrm{e}]$ stars. The selection criteria of the RIOTS4 survey only identify luminous, early-type stars. This means that we can immediately rule out the $\mathrm{B}[\mathrm{e}]$ phenomenon due to planetary nebula sources or symbiotic stars. Thus, the stars must either be pre-main-sequence objects or evolved supergiants. At most, about $25 \%$ of massive stars in the SMC form outside clusters (Oey et al. 2004), and therefore the RIOTS4 field star criteria select against the pre-main-sequence 


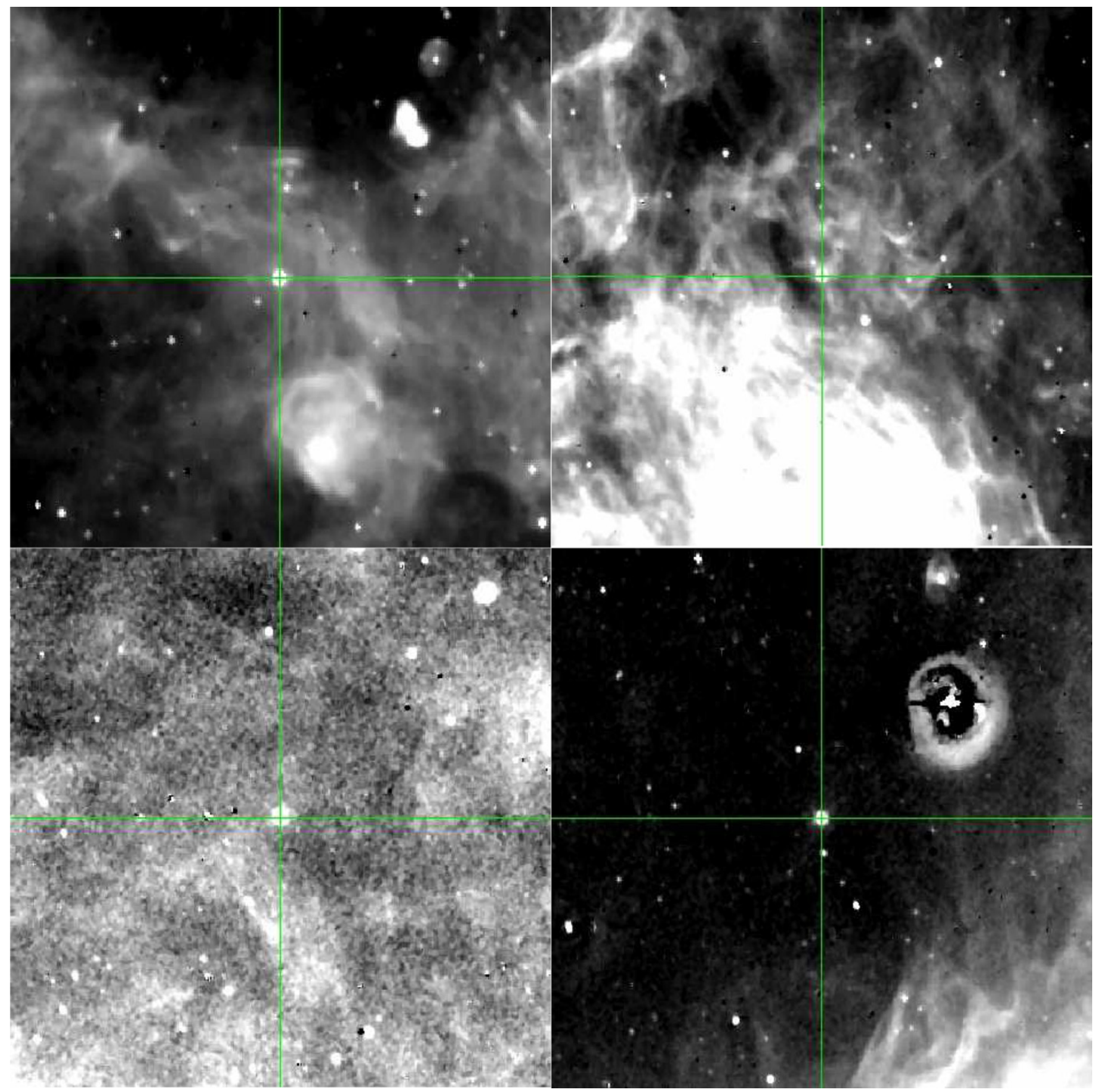

Figure 2. MCELS H $\alpha$ images of the regions around our stars (clockwise, from top left): AzV 154, R15, R38, and R48. The top left image is 116 pc across, the rest are $186.6 \mathrm{pc}$ across.

(A color version of this figure is available in the online journal.)

Herbig B [e] stars. To further verify that our stars are not present in star-forming regions, we searched for dense nebulae by checking images of our stars in $\mathrm{H} \alpha$, [S II], and [O III] using data from the Magellanic Clouds Emission-Line Survey (MCELS; Smith \& MCELS Team 1998; Pellegrini et al. 2012). Figure 2 shows that although there is nebular emission present near R15, none of the stars are inside regions clearly suggestive of current, active star formation.

We then compare our stars to the criteria of $\operatorname{sgB}[\mathrm{e}]$ stars laid out by Lamers et al. (1998). Our stars are all supergiants, having luminosities above the limit suggested by Lamers of $\log \left(L / L_{\odot}\right) \geqslant 4$ (Table 1). AzV 154 and R38 both show P Cygni profiles in the Balmer recombination lines, which is a secondary criterion of $\operatorname{sgB}[\mathrm{e}]$ stars.

We directly compare the optical spectra of our three newly identified $\mathrm{sgB}[\mathrm{e}]$ stars to that of the previously discovered $\mathrm{sgB}[\mathrm{e}]$ star, AzV 154. While the emission lines of AzV 154 have a much higher intensity than for our stars, they show many of the same lines, particularly the $\mathrm{Fe}$ II and [ $\mathrm{Fe}$ II] lines typical of $\operatorname{sgB}[\mathrm{e}]$ stars (Figure 1). Figure 3 shows a color-magnitude diagram (CMD) for our three stars (plus signs), along with $\mathrm{AzV} 154$ (asterisk). The crosses show the other known SMC $\operatorname{sgB}[\mathrm{e}]$ stars from the literature. Figure 3 indicates that our stars are clearly off the main sequence, as shown by the evolutionary tracks of Charbonnel 


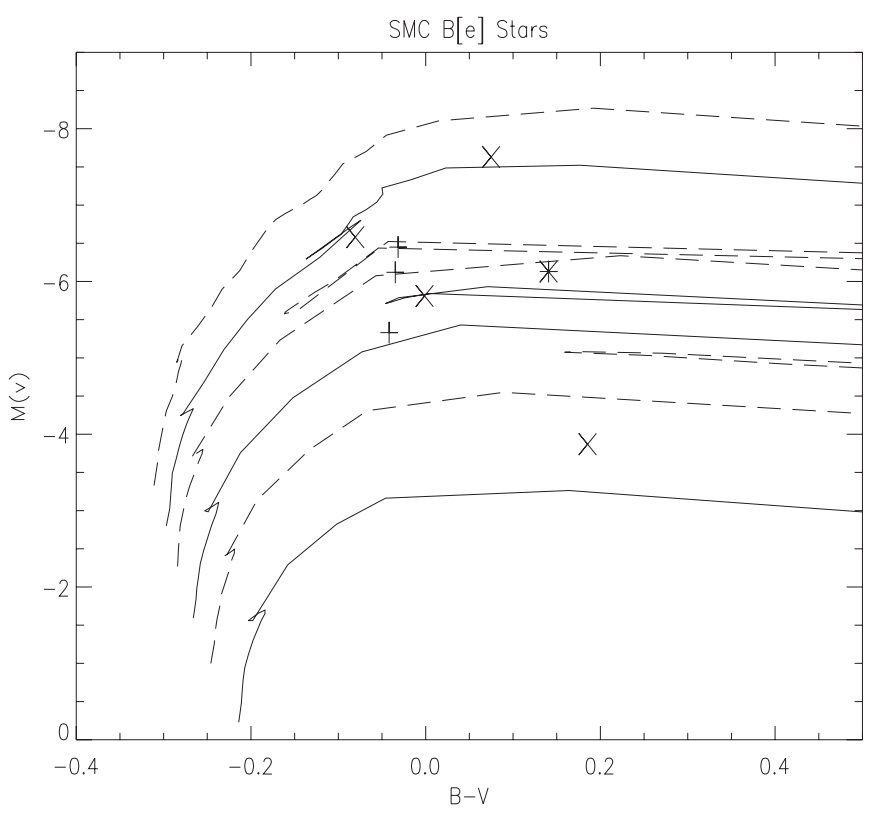

Figure 3. Color-magnitude diagram showing R15, R48, and R38 (plus signs), $\mathrm{AzV} 154$ (asterisk), and the other SMC sgB[e] stars from the literature ( $X$ 's Zickgraf 2006; Wisniewski et al. 2007). The mass tracks were calculated for SMC metallicities of stars (Charbonnel et al. 1993) with masses of 20, 12, and $7 M_{\odot}$ (dashed lines), and 15, 9, and $5 M_{\odot}$ (solid lines).

et al. (1993). The proximity of these other SMC $\operatorname{sgB}[\mathrm{e}]$ stars to our stars suggests that they are near the same evolutionary phase. Thus, our stars are clearly evolved supergiants.

\section{A LACK OF DUST EMISSION}

\subsection{Infrared Data}

An important property of $\mathrm{B}[\mathrm{e}]$ stars is a large infrared excess due to circumstellar dust. $\mathrm{SgB}[\mathrm{e}]$ stars are noted for their combination of high luminosity and strong infrared excess. An example of this is Bonanos et al. (2010) who used data from the Spitzer SAGE SMC survey to study the properties of massive stars in the SMC. In all infrared CMDs and color-color diagrams, the $\mathrm{sgB}[\mathrm{e}]$ stars form a clearly distinct group of massive stars. We obtained $J H K$ photometry of all the B-type stars, including the Be and sgB[e] stars, in the RIOTS4 survey from the Two Micron All Sky Survey (2MASS; Skrutskie et al. 2006), and 3.6-24 $\mu \mathrm{m}$ photometry from the SAGE SMC survey (Gordon et al. 2011). The IR photometry for our objects is given in Table 2 and plotted in Figures 4 and 5 relative to these other groups of SMC B stars. The figures also show the previously known sgB[e] stars in the SMC and LMC (Zickgraf 2006; Wisniewski et al. 2007), which show a large IR excess, whereas our stars are on average 0.6 mag bluer than $\mathrm{AzV} 154$ in $H-K, 0.3 \mathrm{mag}$ bluer in $J-K$, and 2.4 mag bluer in $J-[3.6]$. The modest IR excess for our stars, R15, R38, and R48, is similar to that for classical Be stars. In contrast, B stars that do not show evidence of a circumstellar disk have IR colors around zero, with the exception of some outliers which are just evolving off the main sequence.

Figure 6 shows the infrared spectral energy distributions (SEDs) for the $\operatorname{sgB}[\mathrm{e}]$ stars, using the same data sets. For comparison, we also show the SEDs for three other stars from the RIOTS4 survey including a representative classical Be star, smc 37502 (Massey 2002); and OB supergiant, smc 19728, along with a late-type $\mathrm{B}$ supergiant $(\mathrm{AzV}$ 65) taken from Bonanos

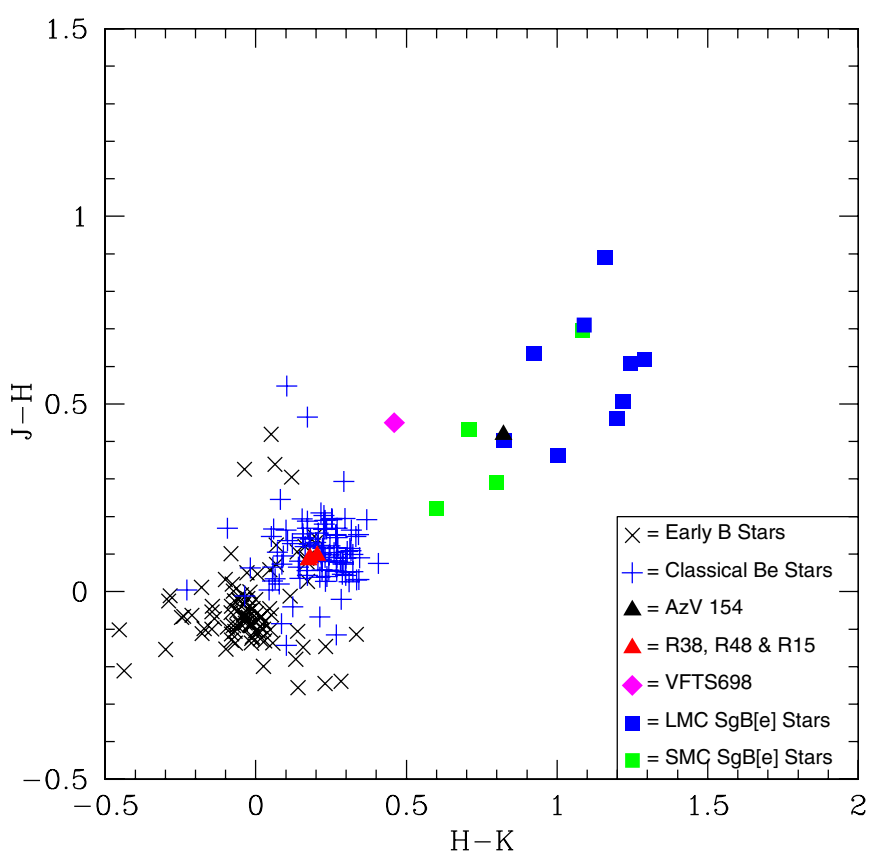

Figure 4. Near-IR color-color diagram $(J-H$ vs. $H-K)$ comparing R15, $\mathrm{R} 48$, and R38 with SMC B and Be stars, and previously known $\mathrm{sgB}[\mathrm{e}]$ stars in the Magellanic Clouds. This figure demonstrates that our new objects show IR excess typical of classical Be stars and atypical of most other $\mathrm{sgB}[\mathrm{e}]$ stars in both the LMC and the SMC.

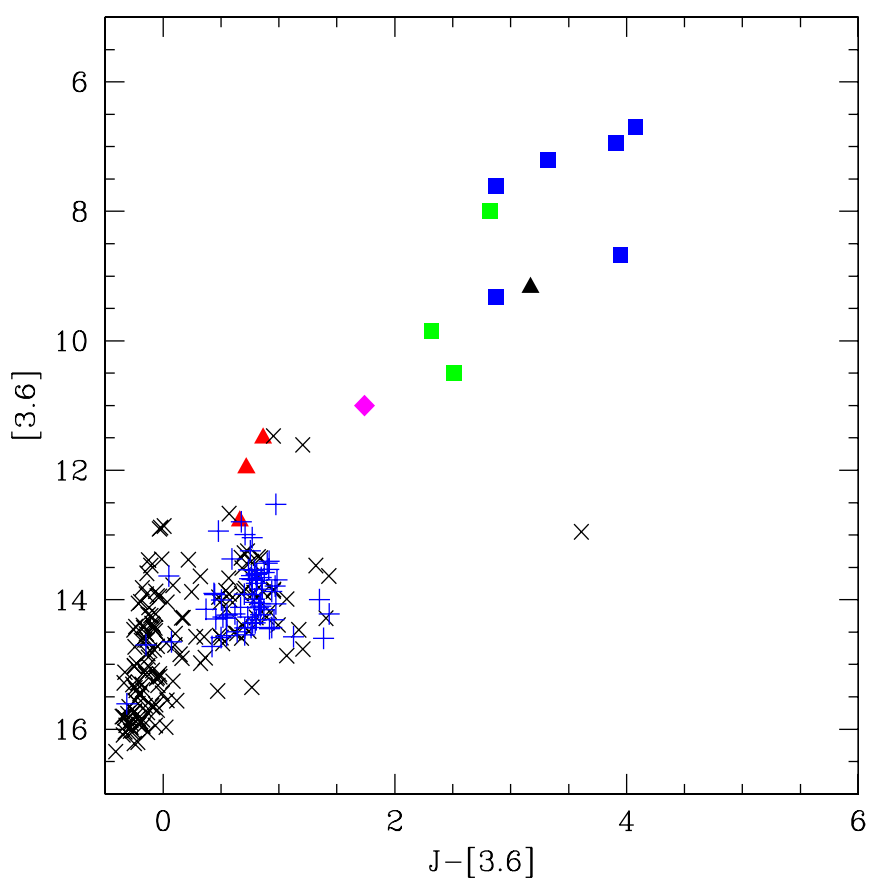

Figure 5. Color-magnitude diagram of [3.6] vs. $J-[3.6]$, again showing the disparity between our stars and known $\mathrm{sgB}[\mathrm{e}]$ stars. The [3.6] mag for the LMC stars are adjusted to the SMC distance by the 0.4 mag difference in their distance moduli. Symbols are the same as in Figure 4.

et al. (2010). Again, our three stars are very clearly different from the previously known sgB[e] star, AzV 154. However, they are also different from the B supergiants, whose SEDs are steeper. Interestingly, the infrared properties of our three stars closely match the infrared properties of classical Be stars. This is apparent in all of Figures 4-6. 


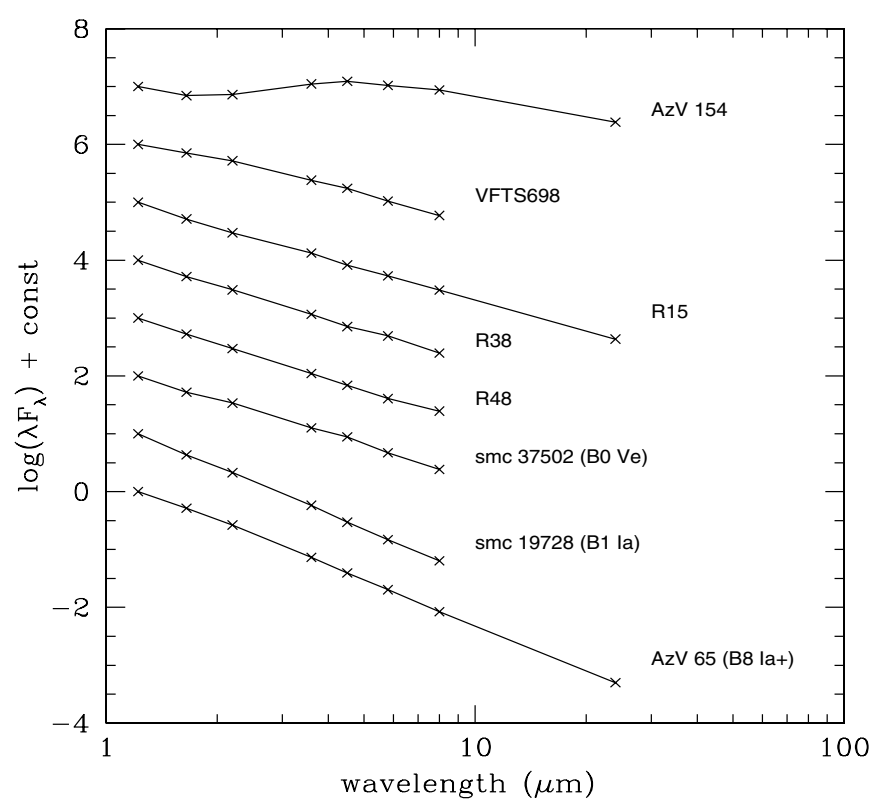

Figure 6. IR SEDs of R15, R38, R48, and AzV154, together with that for VFTS 698 , a dust-poor $\operatorname{sgB}[\mathrm{e}]$ star in the LMC. The SEDs are normalized to their $J$-band magnitudes. For comparison, we also show a classical B0 Ve star (smc 37502), a B1a supergiant (smc 19728), and a late B8Ia+ supergiant (AzV 65; Bonanos et al. 2010).

\subsection{Low Infrared Excess}

Through the infrared data obtained from the 2MASS and SAGE SMC surveys, we see that our stars show an infrared excess that is distinctly lower than that of previously discovered $\mathrm{sgB}[\mathrm{e}]$ stars. This suggests a lack of warm dust in the circumstellar material around our three stars. An excess in $K-[24] \geqslant$ 5 indicates the presence of an optically thick, dusty disk (Hernández et al. 2006). Our stars fall well below that limit, around $K-[24]=2.8$, indicating that a dusty circumstellar disk is lacking, but indicating the presence of an optically thin circumstellar disk, which is characterized by $K-[24] \geqslant 0.3$.

The modest infrared excess of our stars is similar to that of classical Be stars (Figures 4 and 5), and their IR SEDs are also similar (Figure 6). Figure 6 shows that R15, R38, and R48 lack the IR bump from hot dust emission typical of $\mathrm{sgB}$ [e] stars like $\mathrm{AzV}$ 154. While our stars are much brighter, being supergiants, the general shape of the SEDs of our stars more closely follows that of classical Be stars. Thus, the IR emission in our objects may not be due to dust emission as is typical of $\operatorname{sgB}[\mathrm{e}]$ stars, but may instead result from free-free emission as in classical $\mathrm{Be}$ stars (Gehrz et al. 1974). In the IR, free-free opacity increases at longer wavelengths, leading to an inverse power-law SED (Lamers \& Cassinelli 1999). For example, Kastner et al. (2010) obtained IR spectra of several $\mathrm{sgB}[\mathrm{e}]$ stars and found evidence of free-free emission in the near-IR. Thus our stars appear to have optically thin circumstellar disks, since they show weak IR excesses.

The lack of circumstellar dust could be due to a couple of different factors. First, the circumstellar disks around our stars could be ultra metal-poor. The SMC is already known to be a metal-poor environment, and the normal sgB[e] stars in the SMC show a lower infrared excess on average than those in either the Large Magellanic Cloud (LMC) or the Galaxy. Alternatively, our $\mathrm{sgB}[\mathrm{e}]$ stars simply could have much smaller, or less massive, circumstellar disks, possibly because they are just transitioning into or out of the $\operatorname{sgB}[\mathrm{e}]$ phase. If there is a lack of material in the disks around our stars, then the disks would be optically thin, meaning that dust would not be able to form, and the only infrared excess would be due to free-free emission. If our stars have such low-mass circumstellar disks, this would manifest in the optical spectra through the presence of weak emission lines, as opposed to the very strong emission lines seen in other $\mathrm{sgB}[\mathrm{e}]$ stars; this is exactly what is seen in our spectra.

\subsection{A New Class of sgB[e] Stars?}

We have referred to R15, R38, and R48 as $\mathrm{sgB}[\mathrm{e}]$ stars in this work. However, as described earlier, the current definition of B[e] stars includes a "strong" infrared excess (e.g., Conti 1997; Zickgraf 1998). This has been straightforward, since the optical spectral properties and infrared emission have always appeared to occur together. Now, however, our discovery of several objects having optical $\mathrm{B}[\mathrm{e}]$ spectra, while lacking the strong IR excess, presents a complication for classification. While Lamers et al. (1998) specify the criteria for B[e] stars to include the strong IR excess, he also states that the spectroscopic criteria were defined for the optical spectrum. On the advice of Peter Conti and Henny Lamers, we classify these IR-weak objects as B[e] stars, based on the original, optically based definition of the stars (P. Conti 2012, private communication; H. J. G. L. M. Lamers 2012, private communication). Our classification therefore reverts to a definition of $\mathrm{B}[\mathrm{e}]$ stars based exclusively on the existence of B-star properties together with optical permitted and forbidden emission lines (Conti 1976). This is reinforced by the fact that the $\mathrm{B}[\mathrm{e}]$ nomenclature references only the optical spectral features.

Our three objects have optical and infrared properties that are quantitatively more similar to each other than to other $\operatorname{sgB}[\mathrm{e}]$ stars. This is not the first detection of objects showing all of the properties of $\mathrm{sgB}[\mathrm{e}]$ stars, but lacking the characteristic dusty infrared excess. Recently, Dunstall et al. (2012) reported the detection of a similar object in 30 Doradus in the LMC. This object, VFTS 698, shows optical [Fe II] emission and IR properties similar to the three stars that we have found, and to classical Be stars. As also found in our stars, the photospheric emission is visible in VFTS 698, as seen in the absorptionline spectrum of $\mathrm{He} \mathrm{I}$ and $\mathrm{Si}$ II, whereas the photosphere is only occasionally seen in $\mathrm{sgB}[\mathrm{e}]$ stars. We include the data for this star in Table 2 and Figures 4-6. There are likely to be a number of other such known stars, for example, MWC 314 in the Milky Way (Miroshnichenko 1996; Miroshnichenko et al. 1998). We caution that both VFTS 698 and MWC 314 are known binaries, which may complicate the comparison with our newly found SMC objects. However, one of our objects, R48, shows an anomalously high RV (Table 1) which may be evidence of binarity. Thus, dust-poor $\mathrm{sgB}[\mathrm{e}]$ stars are found in widely different environments: VFTS698 in 30 Doradus, our three stars in the field of the SMC, and MWC 314 in the Milky Way. We therefore propose that such stars may be a distinct subset of $\mathrm{sgB}[\mathrm{e}]$ stars, which may be transitioning to or from the full-fledged $\mathrm{B}[\mathrm{e}]$ phenomenon.

\section{CONCLUSIONS}

We report spectroscopic observations of three new $\operatorname{sgB}[\mathrm{e}]$ stars, along with similar observations of $\mathrm{AzV}$ 154, a previously known $\operatorname{sgB}[\mathrm{e}]$ star. These stars were observed in the course of the RIOTS4 survey of massive field stars in the SMC. Our stars show rich emission-line spectra, including emission in the Balmer lines, and forbidden and permitted emission from 
low-excitation metals typical of the $\mathrm{B}[\mathrm{e}]$ phenomenon. The stars have supergiant luminosities. Based on observations of our stars in $\mathrm{H} \alpha,[\mathrm{S} I \mathrm{II}$, and [O III], they are apparently not associated with star-forming regions, implying that they are unlikely to be Herbig B[e] stars. The presence of $\mathrm{P}$ Cygni profiles in one of the spectra, the comparison to $\mathrm{AzV} 154$, and the position of our stars on the H-R diagram are also consistent with the properties of $\operatorname{sgB}[\mathrm{e}]$ stars.

Most interestingly, our stars do not show the strong IR excess characteristic of other $\mathrm{sgB}[\mathrm{e}]$ stars. Instead, the IR emission from our stars resembles that of classical Be stars, suggesting that it originates from free-free emission. Thus, our stars lack the strong circumstellar dust disks that produce the IR excess in $\mathrm{B}[\mathrm{e}]$ stars. This could be due to low dust content in the disks, or it could be due to the disks being of much lower mass. The existence of other dust-poor sgB[e] stars (e.g., Dunstall et al. 2012; Miroshnichenko et al. 1998) suggests that such objects are a distinct sub-category of $\mathrm{sgB}[\mathrm{e}] \mathrm{stars}$, which may be transitioning toward or away from the normal $\mathrm{sgB}[\mathrm{e}]$ phase.

We thank Nuria Calvet, Peter Conti, Joel Kastner, Henny Lamers, and Selma de Mink for useful discussions. We are also grateful to the anonymous referee and to Alceste Bonanos for comments on the manuscript. Thanks to Eric Pellegrini for providing nebular data and to Jordan Zastrow for editorial help. Funding for this project was provided by NSF grant AST-0907758.

\section{REFERENCES}

Allen, D. A., \& Swings, J. P. 1976, A\&A, 47, 293

Andrillat, Y., Jaschek, C., \& Jaschek, M. 1997, A\&AS, 124, 441

Azzopardi, M., Vigneau, J., \& Macquet, M. 1975, A\&AS, 22, 285

Battinelli, P. 1991, A\&A, 244, 69

Bonanos, A. Z., Lennon, D. J., Köhlinger, F., et al. 2010, AJ, 140, 416

Charbonnel, C., Meynet, G., Maeder, A., Schaller, G., \& Schaerer, D. 1993, A\&AS, 101, 415

Conti, P. S. 1976, in IAU Symp. 70, General Discussion in Be and Shell Stars, ed. A. Slettebak (Dordrecht: IAU), 447

Conti, P. S. 1997, in ASP Conf. Ser. 120, Luminous Blue Variables: Massive Stars in Transition, ed. A. Nota \& H. J. H. L. M. Lamers (San Francisco, CA: ASP), 161

Drilling, J. S., \& Landolt, A. U. 2000, in Allen's Astrophysical Quantities, ed. A. N. Cox (4th ed.; New York: AIP), 389

Dunstall, P. R., Fraser, M., Clark, J. S., et al. 2012, A\&A, 542, 50

Feast, M. W., Thackeray, A. D., \& Wesselink, A. J. 1960, MNRAS, 121, 337

Fitzpatrick, E. L. 1991, PASP, 103, 1123
Gehrz, R. D., Hackwell, J. A., \& Jones, T. W. 1974, ApJ, 191, 675

Geisel, S. L. 1970, ApJ, 161, 105

Gordon, K. D., Meixner, M., Meade, M. R., et al. 2011, AJ, 142, 102

Harries, T. J., Hilditch, R. W., \& Howarth, I. D. 2003, MNRAS, 339, 157

Hernández, J., Briceño, C., Calvet, N., et al. 2006, ApJ, 652, 472

Jaschek, C., \& Andrillat, Y. 1998, A\&AS, 128, 475

Jaschek, C., Andrillat, Y., \& Jaschek, M. 1996a, A\&AS, 117, 281

Jaschek, M., Andrillat, Y., \& Jaschek, C. 1996b, A\&AS, 120, 99

Kastner, J. H., Buchanan, C., Sahai, R., Forrest, W. J., \& Sargent, B. A. 2010, AJ, 139, 1993

Kraus, S., Calvet, N., Hartmann, L., et al. 2012, ApJ, 746, L2

Lamb, J. B., Oey, M. S., Graus, A. S., \& Segura-Cox, D. M. 2011, in Four Decades of Research on Massive Stars, ed. L. Drissen, C. Robert, \& N. St-Louis (San Francisco, CA: ASP), in press (arXiv:1109.6655)

Lamers, H. J. G. L. M. 2006, in ASP Conf. Ser. 355, Stars with the B[e] Phenomenon, ed. M. Kraus \& A. S. Miroshnichenko (San Francisco, CA: ASP), 371

Lamers, H. J. G. L. M., \& Cassinelli, J. P. 1999, Introduction to Stellar Winds (Cambridge: Cambridge Univ. Press)

Lamers, H. J. G. L. M., Zickgraf, F.-J., de Winter, D., Houziaux, L., \& Zorec, J. 1998, A\&A, 340, 117

Massey, P. 2002, ApJS, 141, 81

Miroshnichenko, A. S. 1996, A\&A, 312, 941

Miroshnichenko, A. S., Fremat, Y., Houziaux, L., et al. 1998, A\&AS, 131,469

Miroshnichenko, A. S., Levato, H., Bjorkman, K. S., \& Grosso, M. 2003, A\&A, 406, 673

Oey, M. S., King, N. L., \& Parker, J. W. 2004, AJ, 127, 1632

Oey, M. S., \& Lamb, J. B. 2011, in Four Decades of Research on Massive Stars, ed. L. Drissen, C. Robert, \& N. St-Louis (San Francisco, CA: ASP), in press (arXiv:1109.0759)

Pasquali, A., Nota, A., Langer, N., Schulte-Ladbeck, R. E., \& Clampin, M. 2000, AJ, 119, 1352

Pellegrini, E. W., Oey, M. S., Winkler, P. F., Points, S. D., \& Smith, R. C. 2012, ApJ, 755, 40

Pojmanski, G. 1997, Acta Astron., 47, 467

Simón-Díaz, S., \& Herrero, A. 2007, A\&A, 468, 1063

Skrutskie, M. F., Cutri, R. M., Stiening, R., et al. 2006, AJ, 131, 1163

Smith, R. C., \& MCELS Team. 1998, PASA, 15, 163

Stee, P., \& de Araujo, F. X. 1994, A\&A, 292, 221

Walborn, N. R., \& Fitzpatrick, E. L. 1990, PASP, 102, 379

Wheelwright, H. E., de Wit, W. J., Oudmaijer, R. D., \& Vink, J. S. 2012, A\&A, 538, A6

Wisniewski, J. P., Bjorkman, K. S., Bjorkman, J. E., \& Clampin, M. 2007, ApJ, 670,1331

Zaritsky, D., Harris, J., Thompson, I. B., Grebel, E. K., \& Massey, P. 2002, AJ, 123,855

Zickgraf, F.-J. 1998, in B[e] stars, ed. A. M. Hubert \& C. Jaschek (Dordrecht: Kluwer), 1

Zickgraf, F.-J. 2006, in ASP Conf. Ser. 355, Stars with the B[e] Phenomenon, ed. M. Kraus \& A. S. Miroshnichenko (San Francisco, CA: ASP), 135

Zickgraf, F.-J., Wolf, B., Stahl, O., \& Humphreys, R. M. 1989, A\&A, 220, 206

Zickgraf, F.-J., Wolf, B., Stahl, O., Leitherer, C., \& Klare, G. 1985, A\&A, 143, 421 